\title{
Transepidermal delivery of cosmeceuticals using radiofrequency and ultrasound: Study of the penetration of a cosmetic gel in vivo by fluorescence microscopy
}

\author{
Mario A Trelles ${ }^{1 *}$, Justo M. Alcolea ${ }^{1,2}$ and Pedro A Martínez-Carpio ${ }^{1,3}$ \\ ${ }^{1}$ Instituto Médico Vilafortuny. Cambrils. Tarragona, Spain \\ ${ }^{2}$ Clínica Alcolea, Barcelona, Spain \\ ${ }^{3}$ IMC-Investiláser, Sabadell, Barcelona, Spain
}

\begin{abstract}
Background: A prospective, controlled study was carried out to determine the transepidermal penetration of a cosmeceutical after permeabilizing the skin using a device which combines radiofrequency and ultrasound.

Material and methods: The Legato II device (Alma Lasers) was used to introduce the fluorescein-stained Serum/cream cosmeceutical gel into the dermis. The treatments were performed in the retroauricular area in 16 patients, and biopsies were taken at 10 minutes and at 15 hours after the procedure. The intensity of dermal fluorescence in the treated samples was compared to that of Autofluorescence Controls (AC) and Technical Controls (TC).

Results: The samples treated with the Legato II device displayed a greater intensity of fluorescence than the AC and TC, both at 10 minutes and 15 hours. The increases in fluorescence were graded as moderate or intense, but in no case as nil or slight. The results at 10 minutes were: Legato II (55.4 \pm 10.1 ), AC (8.6 \pm 2.8 ), TC $(8.2 \pm 3.6)$. At 15 hours, the results were: Legato II $(54.2 \pm 7.2)$, AC $(8.9 \pm 1.7)$, TC $(8.3 \pm 2.4)$. The differences between the samples and the controls were significant, both at 10 minutes and at 15 hours $(\mathrm{p}<0.0008)$.
\end{abstract}

Conclusion: The transepidermal delivery procedure carried out facilitated a prolonged and effective dermal penetration of the topically applied products.

\section{Introduction}

The skin's stratum corneum acts simultaneously as a barrier and as a reservoir for topically applied substances. Topically applied drugs are mainly found in the uppermost $20 \%$ of the stratum corneum. The limiting factor for percutaneous absorption of a drug is the reservoir and penetration into the stratum corneum $[1,2]$. Therefore, penetration enhancement is an important task for modern dermatopharmacology [3-5].

A number of studies dealing with the use of the Legato II II device have appeared recently. This device was designed to cause thermal damage and fractional ablation to the tissue, while allowing for the introduction of drugs or cosmeceuticals into the dermis [6-10]. This method consists of the creation of microchannels, which theoretically cross the Dermo-Epidermal Junction, and through which the introduction of topically applied substances can be forced by means of ultrasound. These microchannels can be created using a fractional $\mathrm{CO}_{2}$ laser [9] or radiofrequency [10]. Immediately after, ultrasound is used to permeabilize the channels and force the topically applied products through them to the interior of the dermis [6-10].

Our experience has proven the clinical efficacy of this method for facial rejuvenation and for reducing acne scarring $[9,10]$. Other authors have achieved excellent results in treating hypertrophic scars and stretch marks [6-8]. Nonetheless, it would seem that this therapeutic effect is primarily due to the radiofrequency applied, rather than the effects of the drugs or molecules that in theory have been delivered into the skin. It has been considered that using this method may involve an effective penetration of platelet-rich plasma [6], retinoic acid [7], triamcinolone [8] and cosmeceuticals $[9,10]$, but, to date, there has been no evidence of these products reaching the dermis.

The aim of this study is to test the possible penetration of a fluorescein-stained cosmeceutical gel, following the recommended treatment protocol for reducing acne scarring, using the Legato II device [10].

\section{Material and methods}

\section{Patients, study design and methodology}

16 patients ( 9 female and 7 male) were treated, voluntarily agreeing to take part in the study by signing the corresponding informed consent. The prospective, controlled study was approved by the corresponding Ethics Committee.

The transepidermal delivery procedure was carried out in vivo on a surface of approximately $2 \mathrm{~cm} \times 1.5 \mathrm{~cm}$ on the right retroauricular

Correspondence to: Mario A Trelles MD,PhD., Instituto Médico Vilafortuny, Fundación Antoni de Gimbernat, Avda. Vilafortuny, 31. 43850 Cambrils, Tarragona, Spain; E-mail: imv@laser-spain.com

Key words: radiofrequency, ultrasound, cosmeceutical, transepidermal delivery, fluorescein, fluorescence

Received: May 11, 2015; Accepted: June 22, 2015; Published: June 25, 2015 

microscopy

area, while the left retroauricular areas were used for the controls. The topically applied cosmeceutical gel was stained with fluorescein to verify its penetration. For the in vitro studies, ellipsoidal samples $(15 \mathrm{~mm} \times 10 \mathrm{~mm})$ of the treated retroauricular skin were taken.

Samples and controls from 8 patients were biopsied at 10 minutes after the procedure; in the other 8 patients, the samples and controls were taken at 15 hours. At both 10 minutes and 15 hours, 8 skin samples $(10 \mathrm{~mm} \times 5 \mathrm{~mm})$ treated with the Legato II device, 4 Technical Controls (TC) of identical dimensions (10 $\mathrm{mm} \times 5 \mathrm{~mm})$ and 4 Autofluorescence Controls (AC) were taken, the latter, using a $3 \mathrm{~mm}$ ellipsoidal punch. The Technical Controls (TC) consisted of applying the stained gel to the left retroauricular area, without using the Legato II device, to determine possible fluorescein penetration by diffusion or through the incisions made to extract the samples.

Before the samples and technical controls were biopsied, the skin was thoroughly cleaned with acetone to remove any remaining superficial fluorescein. The samples were fixed immediately after extraction with 3.7\% paraformaldehyde in Phosphate Buffered Saline (PBS).

\section{Transepidermal delivery procedure}

Transepidermal delivery of the stained gel was carried out using the Legato II Skin Barrier Breakthrough System (Alma Lasers), which combines two technologies-fractional ablative micro-plasma Radiofrequency (RF) and Acoustic Pressure Ultrasound (US) - to deliver drugs and bioactive compounds into the dermis. The device uses the Pixel $\mathrm{RF}^{\mathrm{TM}}$ to create microchannels and cause thermal damage and fractional ablation. Each microchannel is on average 80-120 microns in diameter and has a depth of 100-150 microns, depending on the RF power settings. After topical application of the cosmeceutical to the skin, the ultrasound generated by the Impact ${ }^{\mathrm{TM}}$ module facilitates penetration into the dermis. The mode of operation is based on mechanical (acoustic) pressure and torques by propagation of the US wave via the sonotrode to the distal horn and the creation of a "hammering" effect. This extracts the liquid from within the microchannels and forces the components of the gel to be enhanced under the Dermo-Epidermal Junction junction through them.

The Pixel RF ${ }^{\mathrm{TM}}$ was set at a power of $60 \mathrm{~W}$. The treatment settings of the acoustic pressure ultrasound handpiece were $70 \%$ of the ultrasound energy output and $50 \mathrm{~Hz}$ for the ultrasound sonotrode vibration rate. During the treatment, two criss-cross passes of the wheel tip were performed, that is, four passes of the RF multiple unipolar electrodes, creating a multiple dense micro-perforation in the epidermis. After this action was completed, the stained gel was applied, followed by the ultrasound module (Impact ${ }^{\mathrm{TM}}$ ).

\section{Cosmeceutical composition and fluorescein staining}

The Serum/cream cosmeceutical gel (Alma Lasers), suited for skin rejuvenation treatments, was applied. Its composition includes keratolytics (allantoin), lipids (bisabolol), vitamins (C and E) and bioactive peptides (palmitoyl oligopeptide, palmitoyl tetrapeptide-7, SH-Polypeptide-15, tripeptide-1, trifluoroacetyl tripeptide-2, acetyl tetrapeptide-2, palmitoyl hexapeptide-19 and tripeptide-8).

To prepare the compound for application, $5 \mathrm{~mL}$ of the PixelTreat SR suspension was mixed with an equal amount of $100 \mathrm{mg} / \mathrm{mL}$ of fluorescein sodium salt (Sigma Aldrich, St. Louis, USA), in a 50:50 mixture. Both products were mixed using a spatula in a sterile aluminum container. The product was stirred for 5 minutes, keeping it well protected from the light, until a homogenous mixture was obtained.

\section{Fluorescence microscopy}

Histological cuts were prepared to observe and measure the intensity of the fluorescence, solely in the dermis. The samples were analyzed by two expert dermatopathologists, independent and blind to the study.

The first pathologist scored the intensity of dermal fluorescence using fluorescence microscopy images, based on an autofluorescence pattern, which was given a score of 0 - i.e. no increased fluorescence. The increase in the intensity of dermal fluorescence was scored in the following way: 0 (similar to the control), 1 (slight increase), 2 (moderate increase) and 3 (intense increase). A Zeiss Apotome Axiovert 200M microscope (Jena, Germany) with a 10x objective and an AlexaFluor $488 \mathrm{~nm}$ filter was used. The control from patient number 1 was used as the autofluorescence pattern against which the dermal fluorescence from the remaining samples and AC and CT were compared.

The second pathologist graded the dermal fluorescence using a Leica LAS6000 microscope (Wetzlar, Germany) with a 20x objective, a fluorescence filter (LP515nm) and transmitted light. The Mowiol mounting medium was used. Images were obtained in tiles, and a reconstruction of the sample surface was performed. The marked areas were segmented according to their intensity threshold in contrast with the autofluorescence background. The results were provided as average dermal fluorescence markings, expressed as Arbitrary Linear Units of Fluorescence Intensity (a.l.u.f.i.).

\section{Statistical analysis}

The results are presented as means $(\mathrm{m}) \pm$ standard deviation (SD). The Mann-Whitney $U$ test was used to compare the mean fluorescence values between the samples and the controls. The calculations were performed using SPSS v.13.0 for Windows, and statistical significance was established for values of $\mathrm{p}<0.05$.

\section{Results}

All the samples treated using the Legato II device and viewed under a Zeiss ApotomeAxiovert 200M microscope showed a greater intensity of dermal fluorescence in relation to the autofluorescence and technical controls, both at 10 minutes (Table 1) and at 15 hours (Table 2). In all cases, the increase in fluorescence in relation to the control groups was graded as moderate or intense, and in no cases as nil or slight. All autofluorescence and technical controls showed a dermal fluorescence similar to the autofluorescence pattern. No differences were observed in the samples taken at 10 minutes and at 15 hours. Figures 1-3 show

Table 1. Results at 10 minutes after Legato II treatment. Increase in the intensity of dermal fluorescence with respect to the autofluorescence pattern. $(0=$ no increase, $1=$ slight increase $2=$ moderate increase, $3=$ intense increase). AC: autofluorescence control, TC: technical control.

\begin{tabular}{|c|c|c|}
\hline Patient & Legato II & Control \\
\hline 1 & 2 & $0(\mathrm{AC})$ \\
\hline 2 & 3 & $0(\mathrm{AC})$ \\
\hline 3 & 3 & $0(\mathrm{AC})$ \\
\hline 4 & 3 & $0(\mathrm{AC})$ \\
\hline 5 & 2 & $0(\mathrm{TC})$ \\
\hline 6 & 3 & $0(\mathrm{TC})$ \\
\hline 7 & 3 & $0(\mathrm{TC})$ \\
\hline 8 & 3 & $0(\mathrm{TC})$ \\
\hline
\end{tabular}


Table 2. Results at 15 hours after Legato II treatment. Increase in the intensity of dermal fluorescence with respect to the autofluorescence pattern. $(0=$ no increase, $1=$ slight increase, $2=$ moderate increase, $3=$ intense increase). AC: autofluorescence control, TC: technical control.

\begin{tabular}{|c|c|c|}
\hline Patient & Legato II & Control \\
\hline 9 & 3 & $0(\mathrm{AC})$ \\
\hline 10 & 3 & $0(\mathrm{AC})$ \\
\hline 11 & 2 & $0(\mathrm{AC})$ \\
\hline 12 & 3 & $0(\mathrm{AC})$ \\
\hline 13 & 2 & $0(\mathrm{TC})$ \\
\hline 14 & 3 & $0(\mathrm{TC})$ \\
\hline 15 & 3 & $0(\mathrm{TC})$ \\
\hline 16 & 2 & $0(\mathrm{TC})$ \\
\hline
\end{tabular}
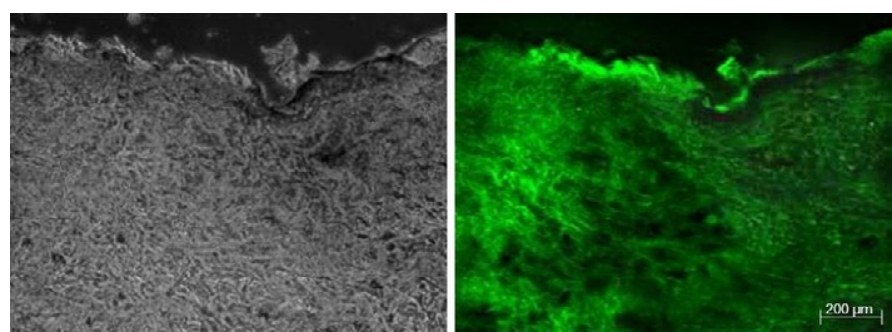

Figure 1: Images corresponding to the Legato II-treated sample from patient 4 . The sample was biopsied at 10 minutes after applying the procedure. The fluorescein is broadly distributed, reaching the deep dermis. The increase in fluorescence with respect to the autofluorescence pattern (Figure 3) was graded as intense.
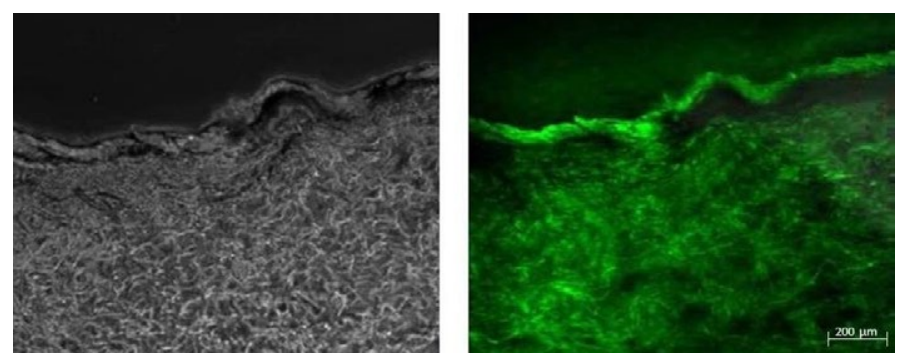

Figure 2: Images corresponding to the Legato II-treated sample from patient 10 . The sample was biopsied at 15 hours after applying the procedure. The fluorescein is broadly distributed, reaching the deep dermis. The increase in fluorescence with respect to the autofluorescence pattern (Figure 3) was graded as intense.
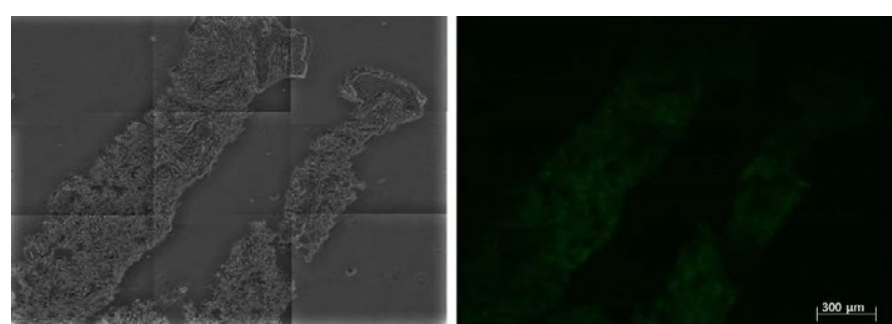

Figure 3: Autofluorescence Control (AC) corresponding to patient 1, which was considered as the autofluorescence pattern from which the fluorescence obtained from the samples, the technical controls and the rest of the AC were compared.

the increase in dermal fluorescence observed in some of the more representative samples in comparison to the autofluorescence pattern.

Tables 3 and 4 show the average dermal fluorescence markings in the treated samples and the controls at 10 minutes and at 15 hours after transepidermal delivery. The values correspond to the means obtained for each sample using a Leica LAS6000 microscope. The average values for all samples (m) and standard deviations (SD) obtained at
10 minutes were: Legato II $(\mathrm{m}=55.4, \mathrm{SD}=10.1), \mathrm{AC}(\mathrm{m}=8.6, \mathrm{SD}=2.8)$, TC $(\mathrm{m}=8.2, \mathrm{SD}=3.6)$. The values obtained at 15 hours were: Legato II $(\mathrm{m}=54.2, \mathrm{SD}=7.2), \mathrm{AC}(\mathrm{m}=8.9, \mathrm{SD}=1.7), \mathrm{TC}(\mathrm{m}=8.3, \mathrm{SD}=2.4)$. The differences between the samples and the controls were significant, both at 10 minutes and at 15 hours $(\mathrm{p}<0.0008)$; no differences were observed at 10 minutes and at 15 hours in the treated samples $(\mathrm{p}=0.872)$.

\section{Discussion}

Although fluorescein could transdermally penetrate the skin, no topical absorption was observed in the technical control group. On the other hand, the transepidermal delivery procedure carried out is effective in regard to cosmeceutical gel permeation, with a very significant exogenous dermal fluorescence. The specific dermal fluorescence is already noticeable at 10 minutes after the procedure, and remains at steady levels of intensity for at least 15 hours. This exceeds the time needed for most drugs and bioactive products to perform their specific action in a single application, and raises the hypothesis of a possible pharmacological effect lasting several days, depending on the drug administered.

It should be noted that the Legato II method has not been considered for regular transdermal drug administration, but for enhancing skin rejuvenation treatments and reducing acne scarring and stretch marks, after applying a new type of radiofrequency (fractional ablative microplasma radiofrequency). In the treatment of acne scarring, satisfactory clinical results are achieved after four treatment sessions, spaced 2 to 3 weeks apart [10]. This implies that the permeated substances used in each session may act in the dermis at high, sustained concentrations. The limitation of this experiment model is that no specific components of the gel were stained for later tracking, and the gel permeation was only shown by the noticeable increase in externally originated dermal fluorescence.

There has been discussion on the importance that ultrasound applied with Impact ${ }^{\mathrm{TM}}$ technology may have in the penetration of substances following a fractional ablation with a $\mathrm{CO}_{2}$ laser [11]. Sadick states that

Table 3. Average dermal fluorescence values in treated samples and controls at 10 minutes after transepidermal delivery. AC: autofluorescence control, TC: technical control. The results are stated as Arbitrary Linear Units of Fluorescence Intensity (a.l.u.f.i.).

\begin{tabular}{|c|c|c|}
\hline Patient & Legato II & Control \\
\hline 1 & 40.7 & $8.3(\mathrm{AC})$ \\
\hline 2 & 64.5 & $12.5(\mathrm{AC})$ \\
\hline 3 & 46.3 & $5.8(\mathrm{AC})$ \\
\hline 4 & 68.0 & $7.9(\mathrm{AC})$ \\
\hline 5 & 57.8 & $6.2(\mathrm{TC})$ \\
\hline 6 & 51.3 & $13.7(\mathrm{TC})$ \\
\hline 7 & 65.9 & $6.0(\mathrm{TC})$ \\
\hline 8 & 48.8 & $7.1(\mathrm{TC})$ \\
\hline
\end{tabular}

Table 4. Average dermal fluorescence values in treated samples and controls at 15 hours after transepidermal delivery. AC: autofluorescence control, TC: technical control. The results are stated as Arbitrary Linear Units of Fluorescence Intensity (a.1.u.f.i.).

\begin{tabular}{|c|c|c|}
\hline Patient & Legato II & Control \\
\hline 9 & 51.7 & $11.1(\mathrm{AC})$ \\
\hline 10 & 67.3 & $9.5(\mathrm{AC})$ \\
\hline 11 & 55.8 & $7.6(\mathrm{AC})$ \\
\hline 12 & 48.5 & $7.5(\mathrm{AC})$ \\
\hline 13 & 56.0 & $6.2(\mathrm{TC})$ \\
\hline 14 & 42.2 & $9.9(\mathrm{TC})$ \\
\hline 15 & 55.8 & $11.0(\mathrm{TC})$ \\
\hline 16 & 56.6 & $6.3(\mathrm{TC})$ \\
\hline
\end{tabular}



microscopy

both laser and ultrasound can facilitate the entry of drugs through the stratum corneum. In this case, a specific type of radiofrequency is used, instead of a laser, which allows for improved results when used at full power [10]. In this case also, radiofrequency and ultrasound can also have a permeating effect of their own. Bommannan et al. showed that ultrasound can, due to its cavitational effects, temporarily reduce the skin barrier $[11,12]$. The relative role that RF or US may have on the permeating effect has not been analyzed in this study. As previously stated, the action mechanisms of RF and US are completely different, and are used simultaneously to achieve a synergic action. For this reason, it was of interest to us to determine whether this synergic action was truly effective, independently of the importance that either of these two technologies may have on its own.

According to the manufacturer's instructions, the number of passes and the parameters used significantly influence the number and depth of the perforations and the effectiveness of the ultrasound. It should be noted that these treatments were performed at the maximum power that RF allows for $(60 \mathrm{~W})$ and with the settings required to achieve US optimal performance, in accordance with the parameters indicated in this study. Lower power settings or other settings, which may also be efficient or may have a different behavior, were not tested.

There has been some discussion on the risks that could arise from introducing drugs directly into the dermis, at doses intended for topical application [11]. In this case, all gel components are substances with well-known properties, each one catalogued and authorized for cosmeceutical use. No adverse side effects which could be attributed to the use of these cosmeceuticals were observed, neither in this research nor in the previous clinical trials $[9,10]$. Other authors also did not observe any complications from using other topically applied drugs, and their therapeutic effects were very clear [6-8]. Issa et al. considered that the therapeutic effect achieved using triamcinolone on hypertrophic scars was sufficient enough to prove the effective penetration of the drug using the Legato II device [8]. However, in our opinion, a histological demonstration was necessary, which was the aim of this study.

For the first time, it has been shown that the Legato II device can achieve the transepidermal delivery of a product (fluorescein) immersed in a cosmeceutical gel. Fluorescein $\left(\mathrm{C}_{20} \mathrm{H}_{12} \mathrm{O}_{5}\right)$ is a hydrosoluble substance from the xanthine family, with a molecular weight of 332.3 $\mathrm{g} / \mathrm{mol}$, and a greater molecular size than other active ingredients contained in the gel. Although our study did not differentiate the penetration of other gel components besides fluorescein, the size of the perforations and the permeation method should allow for the passage of stained gel microparticles with the other substances, as well as fluorescein. The penetration of the products immersed in the gel sustains the good therapeutic results previously observed in the gel $[9,10]$, as well as in the other molecules and drugs applied to date with the Legato II device [6-8].

It is possible that, in the future, similar technology may be used to transepidermally deliver drugs for other therapeutic uses, or to reach the systemic circulation. These results encourage us to carry out further, more selective research, based on the marking of drugs or specific molecules for later tracking.

\section{References}

1. Rougier A, Dupuis D, Lotte C, Roguet R, Schaefer H (1983) In vivo correlation between stratum corneum reservoir function and percutaneous absorption. J Invest Dermatol 81: 275-278. [Crossref]

2. Otberg N, Grone D, Meyer L, Schanzer S, Hoffmann G, et al. (2008) Water-filtered infrared-A (wIRA) can act as a penetration enhancer for topically applied substances. Ger Med Sci 6: Doc08. [Crossref]

3. Yan K, Todo H, Sugibayashi K (2010) Transdermal drug delivery by in-skin electroporation using a microneedle array. Int J Pharm 397: 77-83. [Crossref]

4. Bai Y, Sachdeva V, Kim H, Friden PM, Banga AK (2014) Transdermal delivery of proteins using a combination of iontophoresis and microporation. Ther Deliv 5: 525 536. [Crossref]

5. Jain A, Jain P, Kurmi J, Jain D, Jain R, et al. (2014) Novel strategies for effective transdermal drug delivery: a review. Crit Rev Ther Drug Carrier Syst 31: 219-272. [Crossref]

6. Suh DH, Lee SJ, Lee JH, Kim HJ, Shin MK, et al. (2012) Treatment of striae distensae combined enhanced penetration platelet rich plasma and ultrasound after plasma fractional radiofrequency. J Cosmet Laser Ther 14: 272-276. [Crossref]

7. Issa MC, de Brito Pereira Kassuga LE, Chrevrand NS, do Nascimiento Barbosa L, Luiz RR, et al. (2013) Transepidermal retinoic acid delivery using ablative fractional radiofrequency associated with acoustic presure ultrasound for strech marks treatment. Lasers Surg Med 45: 81-88. [Crossref]

8. Issa MC, Kassuga LE, Chevrand NS, Pires MT (2013) Topical delivery of triamcinolone via skin pretreated with ablative radiofrequency: a new method in hypertrophic scar treatment. Int J Dermatol 52: 367-370. [Crossref]

9. Trelles MA, Leclère FM, Martínez-Carpio PA (2013) Fractional carbon dioxide laser and acoustic presure ultrasound for transepidermal delivery of cosmeceuticals: a novel method of facial rejuvenation. Aesthetic Plastic Surg 37: 965-972. [Crossref]

10. Trelles MA, Martínez-Carpio PA (2014) Attenuation of acné scars using high power fractional ablative unipolar radiofrequency and ultrasound for transepidermal delivery of bioactive compounds through microchannels. Lasers Surg Med 46: 152-159. [Crossref]

11. Sadick NS (2013) Device-assisted transepidermal delivery of cosmeceuticals: a new way to enhance aesthetic procedures? Aesthetic Plast Surg 37: 973-974. [Crossref]

12. Bommannan D, Menon GK, Okuyama H, Elias PM, Guy RH (1992) Sonophoresis. II Examination of the mechanism(s) of ultrasound-enhanced transdermal drug delivery. Pharm Res 9: 1043-1047. [Crossref]

Copyright: (C2015 Trelles MA. This is an open-access article distributed under the terms of the Creative Commons Attribution License, which permits unrestricted use, distribution, and reproduction in any medium, provided the original author and source are credited. 